\title{
GCU
}

Glasgow Caledonian

University

University for the Common Good

\section{Therapeutic potential of co-enzyme Q10 in retinal diseases}

Zhang, Xun; Tohari, Ali Mohammad; Marcheggiani, Fabio; Zhou, Xinzhi; Reilly, James; Tiano, Luca; Shu, Xinhua

Published in:

Current Medicinal Chemistry

DOI:

$10.2174 / 0929867324666170801100516$

Publication date:

2017

Document Version

Author accepted manuscript

Link to publication in ResearchOnline

Citation for published version (Harvard):

Zhang, X, Tohari, AM, Marcheggiani, F, Zhou, X, Reilly, J, Tiano, L \& Shu, X 2017, 'Therapeutic potential of coenzyme Q10 in retinal diseases', Current Medicinal Chemistry, vol. 24, no. 39, pp. 4329-4339.

https://doi.org/10.2174/0929867324666170801100516

\section{General rights}

Copyright and moral rights for the publications made accessible in the public portal are retained by the authors and/or other copyright owners and it is a condition of accessing publications that users recognise and abide by the legal requirements associated with these rights.

Take down policy

If you believe that this document breaches copyright please view our takedown policy at https://edshare.gcu.ac.uk/id/eprint/5179 for details of how to contact us. 
1 Therapeutic potential of co-enzyme Q10 in retinal diseases

2 Xun Zhang ${ }^{1}$, Ali Mohammad Tohari ${ }^{1}$, Fabio Marcheggiani ${ }^{2}$, Xinzhi Zhou ${ }^{1}$, James Reilly ${ }^{1}$,

3 Luca Tiano $^{2}, \mathrm{Xinhua} \mathrm{Shu}^{1}$

41 Department of Life Sciences, Glasgow Caledonian University, Glasgow, UK

52 Department of Dentistry and Clinical Sciences, Polytechnic University of Marche, Ancona, 6 Italy

7

8 Corresponding authors: Luca Tiano, Department of Dentistry and Clinical Sciences,

9 Polytechnic University of Marche, Ancona, Italy. Email: I.tiano@univpm.it; Xinhua Shu,

10 Department of Life Sciences, Glasgow Caledonian University, Glasgow, United Kingdom.

11 Email: Xinhua.Shu@gcu.ac.uk

12

13

14

15

16

17

18

19

20

21

22

23

24

25

26

27

28

29 


\section{Abstract}

Coenzyme Q10 (CoQ10) plays a critical role in mitochondrial oxidative phosphorylation by serving as an electron carrier in the respiratory electron transport chain. CoQ10 also functions as a lipid-soluble antioxidant by protecting lipids, proteins and DNA damaged by oxidative stress. CoQ10 deficiency has been associated with a number of human diseases including mitochondrial diseases, neurodegenerative disorders, cardiovascular diseases, diabetes, cancer, and with the ageing process. In many of these conditions CoQ10 supplementation therapy has been effective in slowing or reversing pathological changes. Oxidative stress is a major contributory factor in the process of retinal degeneration. In this brief review, we summarize the functions of CoQ10 and highlight its use in the treatment of age-related macular degeneration and glaucoma. In light of these data we propose that CoQ10 could have therapeutic potential for other retinal diseases.

Keywords co-enzyme Q10, oxidative stress, retina, age related macular degeneration, glaucoma, retinitis pigmentosa, diabetic retinopathy, protection 


\section{Introduction}

Coenzyme Q10 (CoQ10), first identified by Crane et al, is a 1,4-benzoquinone-containing molecule with a hydrophobic tail harbouring 10 isoprenyl units (1). CoQ10 is ubiquitously distributed in various tissues and blood and presents in all cell membranes $(2,3)$. It is synthesized in the mitochondrial matrix and at least 12 genes are required for its biosynthesis; mutations in some of these genes have been reported to cause CoQ10 deficiencies (4). CoQ10 exists in more than one state within the body: oxidized (ubiquinone), partially reduced (semiquinone radical) and reduced (ubiquinol) forms (Figure 1A); the ratio of oxidized and reduced forms in various cellular membranes is dependent on the metabolic state of individual cells. Within the inner mitochondrial membrane, the CoQ10 pool is found in two main forms: approximately $30 \%$ is protein bound and principally participates in oxidative phosphorylation; the remainder is not protein-bound and contributes to different functions, the major one being as a lipophilic antioxidant (5). CoQ10 is required for cellular ATP generation by shuttling electrons from complexes I and II to complex III in the mitochondrial respiratory chain (Figure 1 B) (6). The oxidized form of CoQ10 is able to undergo two electron reductions in a reaction involving complex I and complex II, resulting in the formation of ubiquinol: subsequently, electrons are passed to complex III. Typically, tissues that are heavily reliant on oxidative metabolism, such as the myocardium, present a high concentration of CoQ10. It is the only naturally occurring endogenous lipid-soluble antioxidant which, in its reduced and active form ubiquinol, may act as a direct free radical scavenger, inhibiting the oxidation of lipids, proteins and DNA (6) or may act synergistically with other antioxidants, such as vitamin E, regenerating its oxidised form, tocopheryl radical. CoQ10 also demonstrates a regulatory role in the expression of genes involved in cell signalling, metabolism and nutrition transport (7). Moreover, it has been shown to exert an anti-inflammatory effect by reducing LPS-induced secretion of TNF- $\alpha$, possibly via the NFkB1-dependent pathway (8).

CoQ10 deficiency is mainly associated with encephalomyopathy, infantile multisystemic disease, cerebellar ataxia, pure myopathy, and cardiofaciocutaneous syndrome. The causes of CoQ10 deficiencies are primarily due to mutations in ubiquinone biosynthesis genes (COQ2, $P D S S 1$ and 2, and $A D C K 3$ ) or in genes indirectly related to CoQ10 biosynthesis (APTX, $B R A F$, and $E T F D H)$. However, the causes of CoQ10 deficiency still remain unknown in a large number of patients (4). Lowered levels of CoQ10 have been reported in different clinical conditions, including cardiovascular disease, diabetes, cancer, and neurodegenerative disease. More generally, a subliminal deficit of CoQ10 might also be observed in 
paraphysiological states such as ageing: synthesis in human is known to peak around the third decade of life and subsequently decreases with age. Moreover, the use of commonly prescribed drugs that interfere with the mevalonate pathway, such as statins, are known also to impact cellular coenzyme Q10 level. Interestingly, intracellular content of CoQ10 is close to the $\mathrm{Km}$ of the respiratory complexes, implying that even slight variations in the CoQ10 content translate into dramatic changes in the mitochondrial bioenergetics that is known to be a major site of production of reactive oxygen species. Oral CoQ10 therapy has been applied to different forms of CoQ10 deficiency, with resulting significant clinical improvements (4). Oxidative stress plays an important role in the pathogenesis of vascular diseases, diabetes and neurodegenerative disease. Due to its antioxidant properties, CoQ10 supplementation has been beneficial in the treatment of the above diseases. Numerous studies have reported that CoQ10 administration improved cardiovascular function $(2,9,10)$. CoQ10 supplementation in three separate clinical trials of dyslipidemic type 2 diabetic patients showed raised plasma CoQ10 levels, improved endothelial function, and decreased blood pressure and glycosylated haemoglobin (HbA1C) (11-13). CoQ10 has been used in different neurodegenerative diseases, including Parkinson's disease, Huntington's disease, and Alzheimer's disease. CoQ10 supplementation seemed to slow progression of Parkinson's disease $(9,14)$.

CoQ10 is detectable in both choroid and retina, though levels are relative low when compared to other oxygen-demanding tissues $(15,16)$. Similarly to other tissues, the level of CoQ10 in the retina declines with age (15). There is increasing evidence that CoQ10 protects retinal cells in vitro and in vivo, therefore the age-related CoQ10 decrease might exacerbate the risk of retinal disease, while supplementation could have a preventative role. Here we provide an overview of the therapeutic roles of CoQ10 in retinal diseases.

\section{Structure and function of mammalian retina}

The neural retinal is a unique structure, consisting of three major cellular layers (outer nuclear layer, ONL; inner nuclear layer, INL; ganglion cell layer, GCL), separated by synaptic layers (Figure 2) (17). An outer monolayer, the retinal pigment epithelium (RPE), underlies the retina and supports photoreceptor function. ONL contains the light-sensitive photoreceptors, rods and cones. Rods are sensitive to dim light, whereas cones function in bright light and colour vision. In the central retina of primates, there is a small cone-enriched area, the 'macula', which is functionally specialised for high acuity vision. The central pit of the macula is the fovea (Figure 2), which contains only cones and provides the sharpest vision (18). In the retinae of most mammalian species, about $95 \%$ of photoreceptors are rods. 
The adult human retina has about 91 million rods and 5 million cones (18). INL is composed mainly of bipolar cells, although amacrine cells and horizontal cells are also localized in this layer. Bipolar cells receive synaptic input from photoreceptors and are responsible for transmitting the signals to ganglion cells directly or indirectly via amacrine cells. Horizontal cells provide feedback to photoreceptor cells and possibly bipolar cells. Amacrine cells are inhibitory neurons and interact with retinal ganglion cells via their dendritic arbors (17). The ganglion cells have long axons, which form the optic nerve, and are responsible for the transmission of signals from photoreceptors to brain.

The retina has the highest oxygen consumption rate (per gram tissue) in the body, which results in the production of a large amount of reactive oxygen and nitrogen species (RONS) that pose a risk for subsequent cellular damage $(19,20)$. The retina, particularly the macula, is also subjected to high light exposure, making photosensitizing molecules, such as retinoids, vulnerable to light damage. In addition, photoreceptor outer segments are extremely lipid-rich: about $15 \%$ of wet weight content is lipid compared with $1 \%$ of wet weight in other types of cells (21). The photoreceptor outer segments also have a high level of the very-long-chain polyunsaturated fatty acid (PUFA), which is vulnerable to RONS and are easily oxidisable to malondialdehyde (MDA) and 4-hydroxy-2-nonenal (HNE) (20). Cellular systems present several defence lines against oxidative damage. However, oxidative imbalances occurring as a result of the ageing process promote oxidative damage that might contribute to the pathogenesis of retinal diseases.

Clinical data have demonstrated oxidative stress contributes the pathogenesis of retinal diseases. Lower total antioxidant capacity has been reported in aqueous humor and sera from patients with retinitis pigmentosa (RP) (22). In patients with diabetic retinopathy (DR), lipid peroxidation in serum was significantly increased when compared to that of patients with diabetes (but with no retinopathy) and there is positive correlation between lipid peroxidation and disease severity $(23,24)$. Furthermore, patients with proliferative DR have a markedly increased serum MDA level compared to that of non-proliferative DR patients (25). Recently increased oxidative stress level and decreased antioxidant defence have been identified in the sera from patients with primary open angle glaucoma, pseudoexfoliative glaucoma and primary angle-closure glaucoma $(26,27)$. Due to the central role of oxidative stress in the progression of these diseases, antioxidant therapies may play a role in counteracting retinal degeneration. Actually antioxidant supplementation in patients with nonproliferative DR demonstrated retardation of disease progress and maintenance of plasma antioxidant capacity (28). 


\section{Protection of retinal diseases by co-enzyme Q10}

\subsection{Age related macular degeneration}

Age-related macular degeneration (AMD) is the most common cause of blind registration in the developed world (29). Early AMD is characterized by drusen formation and pigmentary changes. Late AMD is characterized by geographic atrophy (dry AMD) and / or choroidal neovascularisation (CNV, wet $\mathrm{AMD})$. Wet AMD presents newly formed immature blood vessels growing from the choroid through Bruch's membrane toward the outer retina. Wet AMD accounts only for about $10-15 \%$ of cases, but for $80-90 \%$ of resultant blindness. AntiVEGF (vascular endothelial growth factor) therapies dramatically halt progression of CNV in the majority of wet AMD patients but there is no effective treatment for AMD patients with geographic atrophy. An important pathological feature of AMD is the accumulation of both focal (drusen) and diffuse extracellular (basal) deposits in the macula, between the retinal pigment epithelium (RPE) and the adjacent Bruch's membrane. These deposits lead to dysfunction and subsequent death of RPE and associated photoreceptors (30). It is well recognized that oxidative damage plays an important role in AMD and that antioxidant supplementation can protect against the condition (31).

Blasi et al. measured CoQ10 in plasma and platelets of 19 patients with exudative AMD and 19 age-matched controls (32). They found that most AMD patients had a lower level of plasma CoQ10 than that of most controls, suggesting a link between CoQ10 level and AMD (32). Fourteen early AMD patients treated with a mixture including polyunsaturated fatty acids (1320 mg/day), acetyl-L-carnitine (500 mg/day), CoQ10 (30 mg/day), and vitamin E (30 mg/day) showed slight improvement in visual functions after three months of treatment; the improved visual functions remained relatively steady until 24 months. By contrast, the visual functions of controls treated with vitamin E only (30 mg/day) slowly worsened (33). The same research group continued to evaluate the treatment efficacy of a combination of acetyl-L-carnitine, n-3 fatty acids and CoQ10 in early AMD patients for 12 months (34). 106 patients were randomly allocated to two groups: the treated group (51 patients) and the placebo group (55 patients); four efficacy parameters including visual field mean defect (VFMD), visual acuity, foveal sensitivity and fundus alteration were measured. The treated group showed significant improvement in visual function, demonstrating a significant difference in VFMD, visual acuity and foveal sensitivity when compared to that of the placebo group. Only $2 \%$ of the treated group exhibited clinically related worsening in VFMD while $17 \%$ of the control group showed further deterioration by the end of the trial (34). 


\subsection{Glaucoma}

197 Glaucoma is a leading cause of irreversible blindness, affecting more than 70 million people worldwide (35). It is characterized by the progressive degeneration of retinal ganglion cells. Intraocular pressure (IOP) is higher in many glaucoma patients and regarded as an important factor for initiating neuronal damage in these patients. Previous studies demonstrated that elevated acute and chronic IOP induced oxidative stress in the retina (36-38), resulting in the oxidative modification of proteins, lipids and DNA (39-41). Primary and secondary hypoxia (the latter subsequent to elevated IOP) result in oxidative stress and glutamate excitotoxicity, both of which contribute to ganglion cell dysfunction in glaucoma (42). Histological studies on glaucomatous eyes from patients and different animal models demonstrated that ganglion cells were degenerated through apoptosis (43-47). The death of ganglion cells is mainly caused by oxidative damage via multiple pathogenic mechanisms $(42,48)$. Antioxidants $(n-3$ PUFAs, $\alpha$-Lipoic acid and mitochondrially-targeted SKQ1) treatment in glaucoma animal models showed protection of retinal ganglion dysfunction (49-52).

CoQ10 has also been used to protect retinal ganglion cell function in the glaucomatous condition. In vitro studies demonstrated that CoQ10 treatment increased survival of RGC-5 cells (a rat ganglion cell line) from apoptosis when exposed to $\mathrm{H}_{2} \mathrm{O}_{2}$, radiation, antimycin (the complex III inhibitor) or serum starvation (53-55). Administration of CoQ10 in high intraocular pressure-induced ischemia rat model prevented ganglion cell loss (56). The protection of ganglion cell death by CoQ10 in ischemic retina was through ameliorating oxidative stress, blocking apoptosis, preserving mitochondrial function and inhibiting microglial activation (57). In untreated mouse ischemic retina, the level of superoxide dismutase 2 (SOD2) and heme oxygenase 1 (HO-1) was significantly increased at $12 \mathrm{~h}$ after transient retinal ischemia when compared to non-ischemic control retina; however, CoQ10 treatment preserved SOD2 and HO-1 at levels similar to those of non-ischemic retina. The level of apoptosis-associated protein Bax was significantly increased in ischemic retina, but CoQ10 treatment markedly decreased Bax expression. In addition, the expression of glial fibrillary acidic protein (GFAP, a marker for astroglial cells) and Iba-1 (a marker for microglial cells) was significantly decreased in CoQ10-treated ischemic retina when compared to that of non-treated ischemic retina, demonstrating the inhibition of astroglial and microglial cell activation (57).

Glutamate excitotoxicity can cause ganglion cell death in glaucoma through the $\mathrm{N}-$ methyl-D-aspartate (NMDA) receptor-activated influx of extracellular calcium into cells, which regulates the activities of cell-death-associated enzymes $(42,58)$. Significantly 
increased retinal extracellular glutamate was detected in pressure-induced ischemic rat model; intraocular administration of CoQ10 markedly minimized the increase (56). In an intravitreally NMDA-injection-induced retinal damage mouse model, oral administration of $\mathrm{CoQ} 10(10 \mathrm{mg} / \mathrm{kg})$ for 14 days showed that CoQ10 exerted neuroprotective effects by decreasing ganglion cell death significantly when compared to that of untreated ischemic retina (53). When CoQ10 was administered as eye drops on mouse cornea, it reached the choroid/ retina in a dose- and time-dependent manner (55). Moreover, in patients undergoing vitrectomy CoQ10 administered by eye drops has been shown to penetrate the vitreous body, where it could function on the retinas (59). In a retinal damage mouse model made by intravitreal injection with kainite (a glutamate agonist), CoQ10 eye drop treatment significantly reduced ganglion cell death by inhibiting caspase-dependent apoptosis (55). Lee et al investigated the neuroprotective effects of CoQ10 in a glaucoma mouse model (DBA/2J) by feeding the glaucomatous mice with $\mathrm{CoQ} 10$ for 6 months (60). The survival of ganglion cells was markedly increased in the CoQ10 treated mouse retina when compared to that of mouse fed with a control diet. Similar to the data from retinal ischemic mouse model (57), the protection of ganglion cell death by $\mathrm{CoQ} 10$ also resulted from ameliorating glutamate excitotoxicity, blocking oxidative stress, maintaining mitochondrial function and inhibiting astroglial activation (60). Most recently, open-angle glaucoma (OAG) patients treated with eye drops containing CoQ10 and vitamin $\mathrm{E}$ (in addition to a $\beta$-blocker monotherapy) for 12 months showed beneficial effects on function of the inner retina (assessed by pattern electroretinogram) and enhanced visual cortical response (assessed by pattern visual-evoked potential) (61).

Astrocytes are the major type of glial cell in the optic nerve head $(\mathrm{ONH})$ and provide support for axon function (61). During the progression of glaucoma, astrocytes become activated and are involved in the $\mathrm{ONH}$ remodelling associated with the condition $(62,63)$. Oxidative stress is known to reactivate $\mathrm{ONH}$ astrocytes and is implicated in the pathogenesis of glaucoma $(64,65)$. When rat $\mathrm{ONH}$ astrocytes were treated with $\mathrm{CoQ} 10$ and $\mathrm{H}_{2} \mathrm{O}_{2}$, cell viability and ATP in these cells were both significantly increased, while ROS production was markedly reduced, when compared to that of cells treated with $\mathrm{H}_{2} \mathrm{O}_{2}$ alone. The GFAP, SOD2 and HO-1 proteins in CoQ10 treated cells were significantly decreased compared to those of $\mathrm{H}_{2} \mathrm{O}_{2}$-treated cells. CoQ10 treatment preserved mitochondrial morphology and biogenesis by upregulating the expression of the mitofilin and PGC-1 $\alpha$ proteins, respectively (66). These data suggest that $\mathrm{CoQ} 10$ can protect $\mathrm{ONH}$ astrocytes from oxidative stress mainly through maintenance of mitochondrial function. 


\section{Potential of CoQ10 for the treatment of retinitis pigmentosa and diabetic retinopathy}

As oxidative stress also plays a critical role in the pathogenesis of retinitis pigmentosa and diabetic retinopathy, so CoQ10 has potential for the treatment of both diseases.

\subsection{Retinitis pigmentosa}

268

269

270

271

272

273

Retinitis pigmentosa (RP, MIM \#268000) is a heterogeneous group of conditions involving progressive degeneration of photoreceptor cells and affects $1 / 4000$ individuals worldwide (67). The early clinical feature of RP is night blindness, often starting in adolescence, followed by progressive loss of peripheral vision and late loss of central vision. The characteristically clinical feature is bone spicule pigment deposits presented in the retinal fundus (Figure 3). RP may occur alone, as non-syndromic RP, without other clinical features, or as syndromic RP with different clinical phenotypes. Most RP cases are presumed to result from a mutation in one or more genes and may show autosomal dominant, recessive, Xlinked, or mitochondrial inheritance, although about one-half of all cases are sporadic. Mutations in more than 62 genes have been reported to cause non-syndromic RP, including 23 genes associated with autosomal dominant RP, 36 genes associated with recessive RP, and 3 genes associated with X-linked RP (68).

Death of rod cells in RP occurs through both caspase-dependent and -independent apoptosis, while death of cone cells occurs primarily through necrosis $(69,70)$. Oxidative damage plays a critical role in the death of photoreceptors (69). Photoreceptors have one of the highest rates of oxygen consumption in the body and this is particularly high in the parafoveal region of primates, where rod density is highest (71). Our previous work showed that severe oxidative stress was present in the retinas of four RP mouse models $\left(\operatorname{Pde} 6 b^{\text {rd1/rd1 }}\right.$, Pde $6 b^{\text {atrd } 1 / \text { atrd1, }} \mathrm{Rho}^{-/-}$and Prph $2^{\text {rds/rds }}$ ) evidenced by significantly reduced retinal complex I activities (14-29\% of wildtype) at a stage when significant photoreceptor loss has not yet occurred (72). In RP, oxidative damage is also a major contributing factor to cone death subsequent to the death of rod cells. Further antioxidants have been shown to slow/reduce cone cell death in different RP animal models (73). Upregulation of antioxidant defences by over-expression of both superoxide dismutase 2 (SOD2) and catalase in photoreceptor mitochondria also reduces cone cell death in RP mouse models (74).

It is desirable to develop new candidates with the potential of reducing reactive oxygen species (ROS) production and/or upregulating antioxidant defences, which in turn can potentially slow down retinal degeneration in RP.

\subsection{Diabetic retinopathy}


Diabetic retinopathy (DR) refers to the irreversible damage of retinal cells and structures as a result of chronic diabetes. DR is a progressive disease that is influenced by the duration and control of diabetes, and its development is believed to occur gradually with different degrees of disease severity. DR is classified into five stages: no diabetic retinopathy, background diabetic retinopathy, non-proliferative diabetic retinopathy (NPDR), proliferative diabetic retinopathy $(\mathrm{PDR})$ and diabetic macular edema $(\mathrm{DME})(75,76)$. The first stage, no diabetic retinopathy (Figure 4A), is characterized by normal retinal histology with absence of any abnormal neovascularization and microvascular abrasions. The second stage, background DR (Figure 4B), is the earliest stage of DR and is associated with the presence of low grade of microaneurysm, retinal hemorrhage and exudate. The third stage, non-proliferative diabetic retinopathy (NPDR), itself includes three phases: mild NPDR (Figure 4C) which involves microaneurysm; moderate NPDR (Figure 4D) which involves less severe microaneurysm, intraretinal haemorrhage and microvascular occlusion; and severe NPDR (Figure 4E) which is characterized by severe and increased rate of intraretinal haemorrhage, microvascular abnormalities and venous beading. The fourth stage, proliferative diabetic retinopathy (PDR, Figure 4F), is considered to be a severe phase and is defined by retinal ischemia and increased rate of abnormal neovascularization in the retina, optic disc and iris with vitreous or pre-retinal haemorrhage. The fifth stage, diabetic macular edema (DME, Figure 4G), is associated with relatively increased retinal thickness at the centre of the macula, vascular permeability and leakage, hard exudate, breakdown of blood-retina barrier (BRB) and retinal detachment $(75,76)$.

Oxidative stress is a common characteristic of DR secondary to hyperglycemia. Mitochondria are the principal source of energy production. Under normal conditions, mitochondria provide energy through the electron transport chain (ETC) in which oxygen $\left(\mathrm{O}_{2}\right)$ is utilized as the main electron donor and then reduced to ROS to maintain cellular functions; any increase of ROS level is neutralized by a specialized antioxidant defence system (77). Mitochondria are the main source of ROS production during diabetes, and studies have shown that hyperglycemia induces mitochondrial ROS overproduction in response to increased activation of the polyol pathway, AGEs, PKC pathway, hexosamine biosynthesis, and poly (ADP-riobose) polymerase. Under physiological conditions excess ROS is eliminated by specific antioxidant scavengers and balanced by mitochondria maintaining redox (77). Several antioxidant scavengers such as catalase, superoxide dismutases (SODs) and glutathione peroxidases (GPXs) have been reported to be involved in oxidative stress during DR (78-81). Accumulated data from diabetic patients, diabetic animal models and 
high glucose treated cells has revealed that these anti-oxidants may exhibit different gene expression patterns and activity; the activity of catalase, SODs and GPXs were reported to be low in diabetic patients, animal models and high glucose treated cells compared to normal control (78-81). Thus, a therapeutic strategy to directly decrease ROS production and enhance expression of these anti-oxidants will protect the retina from oxidative stress damage during DR.

\section{Conclusion}

Oxidative stress causes damage to protein, lipid and DNA, which results in retinal cell dysfunction and death. Mitochondria are the major source of oxidative stress. CoQ10 functions as an electron carrier in the mitochondrial respiratory chain and as an intracellular antioxidant that offers therapeutic potential for neurodegenerative diseases. Furthermore, due to its antioxidant properties CoQ10 has demonstrated a protective role in the neuroretina by counteracting oxidative stress, inhibiting microglia cell activation and maintaining mitochondrial function. In particular, the role of CoQ10 in modulating mitochondrial permeability transition pore has been linked to its beneficial effects in preventing the glutamate-induced cytotoxicity that may contribute to neural degeneration. CoQ10 topical eye preparation has been shown to be an effective means of delivery to the vitreous cavity and retina. However, until now only oxidised CoQ10 (ubiquinone) has been tested. The recent availability of the stable formation of the reduced and active form of CoQ10 (ubiquinol) might represent a ground-breaking innovation in the field. In fact, cellular metabolism is able to efficiently promote reduction of exogenously provided coenzyme Q10, while the activity of reducing systems declines with age.

In conclusion, a significant body of evidence supports a role for CoQ10 in promoting eye health through inhibiting ROS production and protecting neuroretinal cells from oxidative damages (Figure 5), although further studies are required to evaluate potential beneficial effects of ubiquinol eye-drop treatment for patients with retinal diseases, including AMD, DR, RP and glaucoma, which are major causes of blindness in the world.

\section{Acknowledgement}

Dr Shu's lab is supported by the Rosetrees Trust, the Fight for Sight, the Yorkhill Children's Hospital Charity and the Visual Research Trust. The Royal Society of Edinburgh funded Dr Luca Tiano's visit to Dr Shu's lab at GCU. 


\section{References}

366 [1] Crane FL, Hatefi Y, Lester RL, Widmer C. Isolation of a quinone from beef heart

367 mitochondria. Biochimica et Biophysica Acta 1957, 25: 220-221.

368 [2] Garrido-Maraver J, Cordero MD, Oropesa-Avila, Vega AF, et al. Coenzyme Q10 therapy.

369 Mol Syndromol, 2014, 5: 187-197

370 [3] Turunen M, Olsson J, Dallner G. Metabolism and function of coenzyme Q. Biochim

371 Biophys Acta 2004, 1660: 171-199.

372 [4] Quinzii CM, Lopez LC, Naini A, DiMauro S, Hirano M. Human CoQ10 deficiencies.

373 BioFactors, 2008, 32, 113-118.

374 [5] Bentinger M, Brismar K, Dallner G. The antioxidant role of coenzyme Q. Mitochondrion 375 2007, 7 Suppl, S41-50.

376 [6] Littarru GP, Tiano L. Bioenergetic and antioxidant properties of coenzyme Q10: recent 377 developments. Mol Biotechnol, 2007, 37, 31-37.

378 [7] Groneberg DA, et al. Coenzyme Q10 affects expression of genes involved in cell 273 379 signalling, metabolism and transport in human CaCo-2 cells. Int J Biochem Cell Biol, 2005, $380 \quad 27437,1208-1218$.

381 [8] Schmelzer C, Lindner I, et al. Function of coenzyme Q10 in inflammation and gene 382 expression. BioFactors, 2008, 32, 179-183.

[9] Littarru GP, Tiano L. Clinical aspects of co-enzyme Q10: an update. Nutrition, 2010, 26, $278250-254$.

[10] Langsjoen H, Langsjoen P, Willis R, Folkers K. Usefulness of coenzyme Q10 in clinical cardiology: a long-term study. Mol Aspects Med, 1994, 15 (suppl): S165-S175.

[11] Hodgson JM, Watts GF, Playford DA, Burke V, Croft KD. Coenzyme Q10 improves blood pressure and glycaemic control: a controlled trial with type 2 diabetes. Eur J Clin Nutr, 2002, 56: 1137-1142. improves endothelial dysfunction of the brachial artery in type II diabetes mellitus. Diabetologia, 2002, 45: 420-426.

[13] Playford DA, Watts GF, Croft KD, Burke V. Combined effect of coenzyme Q10 and fenofibrate on forearm microcirculatory function in type 2 diabetes. Atherosclerosis, 2003, 168: 169-179. functional decline. Arch Neurol 2002;59:1541-50. 
401

402

403

404

405

406

407

408

409

410

411

412

413

414

415

416

417

418

419

420

421

422

423

424

425

426

427

428

429

430

431

432

433

[15] Qu J, Kaufman Y, Washington I. Coenzyme Q10 in the human retina. Invest Ophthalmol Vis Sci, 2009, 50: 1814-1818.

[16] Qu J, Ma L, Washington I. Retinal coenzyme Q in the bovine eye. Biofactors, 2011, 37, 393-398.

[17] Masland RH. The neuronal organization of the retina. Neuron, 2012, 76: 266-280.

[18] Oyster CW. The human Eye-structure and function. 1999. Sunderland, MA: Sinauer.

[19] Yu DY, Cringle SJ. Oxygen distribution and consumption within the retina in vascularised and avascular retinas and in animal models of retinal disease. Prog Retin Eye Res, 2001, 20: 175-208.

[20] Wright AF, Chakarova CF, Abd El-Aziz MM, Bhattacharya SS. Photoreceptor degeneration: genetic and mechanistic dissection of a complex trait. Nat Rev Genet, 2010, 11: 273-284.

[21] Whikehart DR. Biochemistry of the Eye 2nd 305 edn (Butterworth Heinemann, 2003).

[22] Martínez-Fernández de la Cámara C, Salom D, Sequedo MD, Hervás D, Marín-Lambíes C, Aller E, Jaijo T, Díaz-Llopis M, Millán JM, Rodrigo R. Altered antioxidant-oxidant status in the aqueous humor and peripheral blood of patients with retinitis pigmentosa. PLoS One. 2013, 8(9):e74223.

[23] Hartnett ME, Stratton RD, Browne RW, Rosner BA, Lanham RJ, Armstrong D. Serum markers of oxidative stress and severity of diabetic retinopathy. Diabetes Care 2000, 23, 234240.

[24] Gupta MM, Chari S. Lipid peroxidation and antioxidant status in patients with diabetic retinopathy. Indian J. Physiol. Pharmacol. 2005, 49, 187-192.

[25] Mancino R, Di Pierro D, Varesi C, Cerulli A, Feraco A, Cedrone C, Pinazo-Duran MD, Coletta M, Nucci C. Lipid peroxidation and total antioxidant capacity in vitreous, aqueous humor, and blood samples from patients with diabetic retinopathy. Mol. Vis. 2011, 17, 12981304.

[26] Erdurmus M, Yagci, R, Atis O, Karadag R, Akbas A, Hepsen IF. Antioxidant status and oxidative stress in primary open angle glaucoma and pseudoexfoliative glaucoma. Curr. Eye Res. 2011, 36, 713-718.

[27] Chang D, Sha Q, Zhang X, Liu P, Rong S, Han T, Pan H. The evaluation of the oxidative stress parameters in patients with primary angle-closure glaucoma. PLoS One 2011, 6, e27218.

[28] Garcia-Medina JJ, Pinazo-Duran MD, Garcia-Medina M, Zanon-Moreno V, PonsVazquez S. A 5-year follow-up of antioxidant supplementation in type 2 diabetic retinopathy. Eur. J. Ophthalmol. 2011, 21, 637-643. 
[29] Friedman DS et al. Prevalence of age-related macular degeneration in the United States. Arch Ophthalmol, 2004, 122, 564-572.

[30] Abdelsalam A et al. Drusen in age-related macular degeneration: pathogenesis, natural course, and laser photocoagulation-induced regression. Surv. Ophthalmol, 1999, 44, 1 -29

[31] Evans J. Antioxidant supplements to prevent or slow down the progression of AMD: a systemic review and meta-analysis. Eye, 2008, 22: 751-760.

[32] Blasi MA, Bovina C, Carella G, Genova ML, Jansen AMA, Lenaz G, Brancato R. Does coenzyme Q10 play a role in opposing oxidative stress in patients with age-related macular degeneration? Ophthalmologica. 2001, 215: 51-54.

[33] Feher J, Papale A, Mannino G, Gualdi L, Balacco Gabrieli C. Mitotropic compounds for the treatment of age-related macular degeneration. Ophthalmologica. 2003, 217(5):351-7.

[34] Feher J, Kovacs B, Kovacs I, Schveoller M, Papale A, Balacco Gabrieli C. Improvement of visual functions and fundus alterations in early age-related macular degeneration treated with a combination of acetylL-carnitine, n-3 fatty acids, and Coenzyme Q10. Ophthalmologica. 2005;219(3):154-66.

[35] Quigley HA, Broman AT. The number of people with glaucoma worldwide in 2010 and 2020. Br J Ophthalmol. 2006; 90(3):262-267.

[36] Muller A, Pietri S, Villain M, Frejaville C, Bonne C, Culcas M. Free radicals in rabbit retina under ocular hyperpressure and functional consequences. Exp Eye Res, 1997, 64, 327 637-643.

[37] Bonne C, Muller A, Villain M. Free radicals in retinal ischemia. Gen Pharmacol, 1998, $30,329275-280$.

[38] Moreno MC, Campanelli J, Sande P, Sanez DA, Keller Sarmiento MI, Rosenstein RE. Retinal oxidative stress induced by high intraocular pressure. Free Radic Bio, 2004, 37, 803812.

[39] Ko ML, Peng PH, Ma MC, Ritch R, Chen CF. Dynamic changes in reactive oxygen species and antioxidant levels in retinas in experimental glaucoma. Free Radic Biol Med, 2005, 39, 365-373.

[40] Tezel G, Yang X, Cai J. Proteomic identification of oxidatively modified retinal proteins in a chronic pressure-induced rat model of glaucoma. Invest Ophthalmol Vis Sci. 2005, 46,3177-3187.

[41] Inman DM, Lambert WS, Calkins DJ, Horner PJ. $\alpha$-Lipoic acid antioxidant treatment limits glaucoma-related retinal ganglion cell death and dysfunction. PLoS One. 2013, 8, 341 e65389. 
[42] Tezel. Oxidative stress in glaucomatous neurodegeneration: mechanisms and consequences. Prog Retinal Eye Res, 2006, 25, 490-513.

470

471

472

473

474

475

476

477

478

479

480

481

482

483

484

485

486

487

488

489

490

491

492

493

494

495

496

497

498

499

500

501

[43] Kerrigan LA, Zack DJ, Quigley HA, Smith SD, Pease ME. TUNEL-positive ganglion cells in human primary open-angle glaucoma. Arch Ophthalmol. 1997, 115(8):1031-5.

[44] Wax MB, Tezel G, Edward PD. Clinical and ocular histopathological findings ina patient with normal-presure glaucoma. Arch Ophthalmol, 1998, 116, 993-1001.

[45] Garcia-Valenzuela E, Shareef S, Walsh J, Sharma SC. Programmed cell death of retinal ganglion cells during experimental glaucoma. Exp Eye Res. 1995, 61, 33-44.

[46] Quigley HA, Nickells RW, Kerrigan LA, Pease ME, Thibault DJ, Zack DJ. Retinal ganglion cell death in experimental glaucoma and after axotomy occurs by apoptosis. Invest Ophthalmol Vis Sci. 1995, 36, 774-86.

[47] Li Y, Schlamp CL, Nickells RW. Experimental induction of retinal ganglion cell death in adult mice. Invest Ophthalmol Vis Sci. 1999, 40,1004-1008.

[48] Levkovitch-Verbin H. Retinal ganglion cell apoptotic pathway in glaucoma: Initiating and downstream mechanisms. Prog Brain Res. 2015, 220:37-57.

[49] Nguyen CT, Vingrys AJ, Bui BV. Dietary omega-3 fatty acids and ganglion cell function. Invest Ophthalmol Vis Sci. 2008, 49, 3586-3594.

[50] Nguyen CT, Vingrys AJ, Bui BV. Dietary $\omega-3$ deficiency and IOP insult are additive risk factors for ganglion cell dysfunction. J Glaucoma. 2013, 22, 269-277.

[51] Inman DM, Lambert WS, Calkins DJ, Horner PJ. $\alpha$-Lipoic acid antioxidant treatment limits glaucoma-related retinal ganglion cell death and dysfunction. PLoS One. 2013, 8, e65389.

[52] Iomdina EN, Khoroshilova-Maslova IP, Robustova OV, Averina OA, Kovaleva NA, Aliev G, Reddy VP, Zamyatnin AA Jr, Skulachev MV, Senin II, Skulachev VP.

Mitochondria-targeted antioxidant SkQ1 reverses glaucomatous lesions in rabbits. Front Biosci (Landmark 367 Ed). 2015, 20:892-901.

[53] Nakajima Y, Inokuchi Y, Nishi M, Shimazawa M, Otsubo K, Hara H. Coenzyme Q10 protects retinal cells against oxidative stress in vitro and in vivo. Brain Res. 2008, 1226, 226233.

[54] Lulli M, Witort E, Papucci L, Torre E, Schiavone N, Dal Monte M, Capaccioli S. Coenzyme Q10 protects retinal cells from apoptosis induced by radiation in vitro and in vivo. J Radiat Res. 2012, 53, 695-703.

[55] Lulli M, Witort E, Papucci L, Torre E, Schipani C, Bergamini C, Dal Monte M, Capaccioli S. Coenzyme Q10 instilled as eye drops on the cornea reaches the retina and 
protects retinal layers from apoptosis in a mouse model of kainate-induced retinal damage. Invest Ophthalmol Vis Sci. 2012, 53(13):8295-302.

[56] Nucci C, Tartaglione R, Cerulli A, Mancino R, Spanò A, Cavaliere F, Rombolà L, Bagetta G, Corasaniti MT, Morrone LA. Retinal damage caused by high intraocular pressureinduced transient ischemia is prevented bycoenzyme Q10 in rat. Int Rev Neurobiol. 2007, 82, 397-406.

[57] Lee D, Kim KY, Shim MS, Kim SY, Ellisman MH, Weinreb RN, Ju WK. Coenzyme Q10 ameliorates oxidative stress and prevents mitochondrial alteration in ischemic retinal injury. Apoptosis. 2014, 19, 603-614.

[58] Sucher NJ, Lipton SA, Dreyer EB. Molecular basis of glutamate toxicity in retinal ganglion cells. Vis Res, 1997, 37, 3483-3493.

[59] Fato R, Bergamini C, Leoni S, Pinna A, Carta F, Cardascia N, Ferrari TM, Sborgia C, Lenaz G. Coenzyme Q10 vitreous levels after administration of coenzyme Q10 eyedrops in patients undergoing vitrectomy. Acta Ophthalmol. 2010, 88, e150-1.

[60] Lee D, Shim MS, Kim KY, Noh YH, Kim H, Kim SY, Weinreb RN, Ju WK. Coenzyme Q10 inhibits glutamate excitotoxicity and oxidative stress-mediated mitochondrial alteration in a mouse model of glaucoma. Invest Ophthalmol Vis Sci. 2014, 55, 993-1005.

[61] Parisi V, Centofanti M, Gandolfi S, Marangoni D, Rossetti L, Tanga L, Tardini M, Traina S, Ungaro N, Vetrugno M, Falsini B. Effects of coenzyme Q10 in conjunction with vitamin $\mathrm{E}$ on retinal-evoked and cortical-evoked responses in patients with open-angle glaucoma. J Glaucoma. 2014, 23, 391-404.

[62] Hernandez MR. The optic nerve head in glaucoma: role of astrocytes in tissue remodeling. Prog Retin Eye Res. 2000,19, 297-321.

[63] Schneider M, Fuchshofer R. The role of astrocytes in optic nerve head fibrosis in glaucoma. Exp Eye Res. 2016,142,49-55.

[64] Malone PE, Hernandez MR. 4-Hydroxynonenal, a product of oxidative stress, leads to an antioxidant response in optic nerve head astrocytes. Exp Eye Res. 2007, 84, 444-454.

[65] Yu AL, Fuchshofer R, Birke M, Kampik A, Bloemendal H, Welge-Lüssen U. Oxidative stress and TGF-beta 2 increase heat shock protein 27 expression in human optic nerve head astrocytes. Invest Ophthalmol Vis Sci. 2008, 49, 5403-5411.

[66] Noh YH, Kim KY, Shim MS, Choi SH, Choi S, Ellisman MH, Weinreb RN, Perkins GA, Ju WK. Inhibition of oxidative stress by coenzyme Q10 increases mitochondrial mass and improves bioenergetic function in optic nerve head astrocytes. Cell Death Dis. 2013, 4, e820. [67] Hartong DT, Berson EL, Dryja TP. Retinitis pigmentosa. Lancet, 2006, 368, 1795-1809. 
[68] Daiger SP, Sullivan LS, Bowne SJ. Genes and mutations causing retinitis pigmentosa.

537 Clin Genet, 2013, 84: 132-141.

538 [69] Bramall AN, Wright AF, Jacobson SG, McInnes RR. The genomic, biochemical, and 539 cellular responses of the retina in inherited photoreceptor degeneration and prospects for the 540 treatment of these disorders. Annual Rev Neurosci, 2010, 33: 441-72

541 [70] Murakami Y, Matsumoto H, Roh M, Suzuki J, Hisatomi T, Ikeda Y, Miller JW, Vavvas

542 DG. Receptor interacting protein kinase mediates necrotic cone but not rod cell death in a 543 mouse model of inherited degeneration. Proc Natl Acad Sci USA, 2012, 109: 14598-14603.

544 [71] Yu DY, Cringle SJ. Retinal degeneration and local oxygen metabolism. Exp Eye Res, $5452005,80,745-51$.

546 [72] Vlachantoni D, Bramall AN, Murphy MP, Taylor RW, Shu X, Tulloch B, Van Veen 547 T, Turnbull DM, McInnes RR, Wright AF. Evidence of severe mitochondrial oxidative stress 548 and a protective effect of low oxygen in mouse models of inherited photoreceptor 549 degeneration. Hum Mol Genet, 2011, 20: 322-335.

550 [73] Komeima K, Rogers BS, Lu L, Campochiaro PA. Antioxidants reduce cone cell death in 551 a model of retinitis pigmentosa. Proc Natl Acad Sci USA, 2006, 103: 11300-11305.

552 [74] Usui S, Komeima K, Lee SY, Jo YJ, Ueno S, Rogers BS, Wu Z, Shen J, Lu L, Oveson 553 BC, Rabinovitch PS, Campochiaro PA. Increased expression of catalase and superoxide 554 dismutase 2 reduces cone cell death in retinitis pigmentosa. 2009, 17(5):778-786.

555 [75] Hooper P, Boucher MC, Cruess A, Dawson KG, Delpero W, Greve M, Kozousek V, 556 Lam WC, Maberley DA. Canadian Ophthalmological Society evidence-based clinical 557 practice guidelines for the management of diabetic retinopathy. Can J Ophthalmol. 2012, 558 47(2 Suppl):S1-30, S31-54.

559 [76] Cheung, N., Mitchell, P. and Wong, T. (2010). Diabetic retinopathy. The Lancet, $560376(9735), 124-136$.

561 [77] Kowluru RA, Mishra M. Oxidative stress, mitochondrial damage and diabetic 562 retinopathy. Biochim Biophys Acta. 2015,1852(11):2474-83.

563 [78] Eshaq RS, Wright WS, Harris NR. Oxygen delivery, consumption, and conversion to 564 reactive oxygen species in experimental models of diabetic retinopathy. Redox Biol. 2014, 2, 565 661-666.

566 [79] El-Bab MF, Zaki NS, Mojaddidi MA, Al-Barry M, El-Beshbishy HA. Diabetic 567 retinopathy is associated with oxidative stress and mitigation of gene expression of 568 antioxidant enzymes. Int J Gen Med. 2013, 6:799-806. 
569 [80] Yokoyama T, Yamane K, Minamoto A, Tsukamoto H, Yamashita H, Izumi S, Hoppe G,

570 Sears JE, Mishima HK. High glucose concentration induces elevated expression of anti-

571 oxidant and proteolytic enzymes in cultured human retinal pigment epithelial cells. Exp Eye

572 Res. 2006, 83, 602-609.

573 [81] Kowluru RA, Atasi L, Ho YS. Role of mitochondrial superoxide dismutase in the 574 development of diabetic retinopathy. Invest Ophthalmol Vis Sci. 2006, 47(4):1594-1599.

575 [82] Raghpathy RH, Patnaik SR, Shu X. Transgenic zebrafish models for understanding

576 retinitis pigmentosa. Clon Transgen, 2013, 2: 2

577 [83] El-Bab MF, Shawky N, Al-Sisi A, Akhtar M. Retinopathy and risk factors in diabetic

578 patients from Al-Madinah Al-Munawarah in the Kingdom of Saudi Arabia. Clin

579 Ophthalmol. 2012, 6, 269-276.

580 [84] Shotliff K, Duncan G. Diabetic retinopathy: summary of grading and management

581 criteria. Pract Diab Int. 2006, 23:418-420.

582

583

584

585

586

587

588

589

590

591

592

593

594

595

596

597

598 


\section{Figure legends}

600 Figure 1 Coenzyme Q10 is a redox existing in the cellular membranes and consisting of a quinone ring and 10-isoprenoid-unit tail. There are three states of Coenzyme Q10: fully oxidized form (ubiquinone), semiquinone (ubisemiquinone) and fully reduced form (ubiquinol) (A). Coenzyme Q10 is soluble in phospholipid bilayer of the inner mitochondrial membrane. It is an essential component of the mitochondrial respiratory chain (B). Ubiquinone can adopt one or two electrons from inner mitochondrial membrane complex I and II, transforming into semiquinone or ubiquinol by Q10 reductases. Then Q10 transfers the electrons to complex III. The electron is then passed to complex IV through cytochrome C. A component of Complex III can convert ubiquinol to ubiquinone to recycle Q10. Figure 2 The structure of the retina. (A) Cross-sectional image of the heathy retina obtained by optical coherence tomography (OCT). Scans were taken with the upper panel showing the 64th scan and the lower panel showing the 256th scan. (B) Histological structure of mouse retina obtained by hematoxylin-eosin staining (left panel) and by immunostaining with 1D4 antibody (labelling the rod outer segments, right panel). GCL, ganglion cell layer; INL, inner nuclear layer; IS, inner segment; IPL, inner plexiform layer; L, lens; NFL, nerve fiber layer; ONL, outer nuclear layer; OS, outer segments; RPE, retinal pigment epithelium.

Figure 3 Bone spicule pigment deposits are present in the fundus of retinitis pigmentosa patient (right side). Fundus of healthy individual is on the left side. Adapted from Raghpathy et al. (Ref 81)

Figure 4 Clinical classfification of diabetic retinopathy (DR) determined by ophthalmoscopy (fundoscopy). (A) Heathy retina. (B) Background DR. (C) Mild non-proliferative diabetic retinopathy (NPDR). (D) Moderate NPDR. (E) Severe NPDR. (F) Proliferative diabetic retinopathy (PDR). (G) Diabetic macular edema (DME). Adapted from El-Bab et al., 2012 (Ref 82) and Shotliff and Duncan, 2006 (Ref 83).

624 Figure 5 Diagram illustrating protection of co-enzyme Q10 (CoQ10) via inhibiting ROS production. CoQ10 (ubiquinol) blocks the production of ROS and subsequently attenuates oxidative damage and inflammation, which reduce death of retinal cells (photoreceptors, retinal pigment epithelium cells and ganglion cells) and delays the progression of retinal diseases (age-related macular degeneration, AMD; diabetic retinopathy, DR; retinitis pigmentosa, RP; glaucoma). 
Figure 1

A
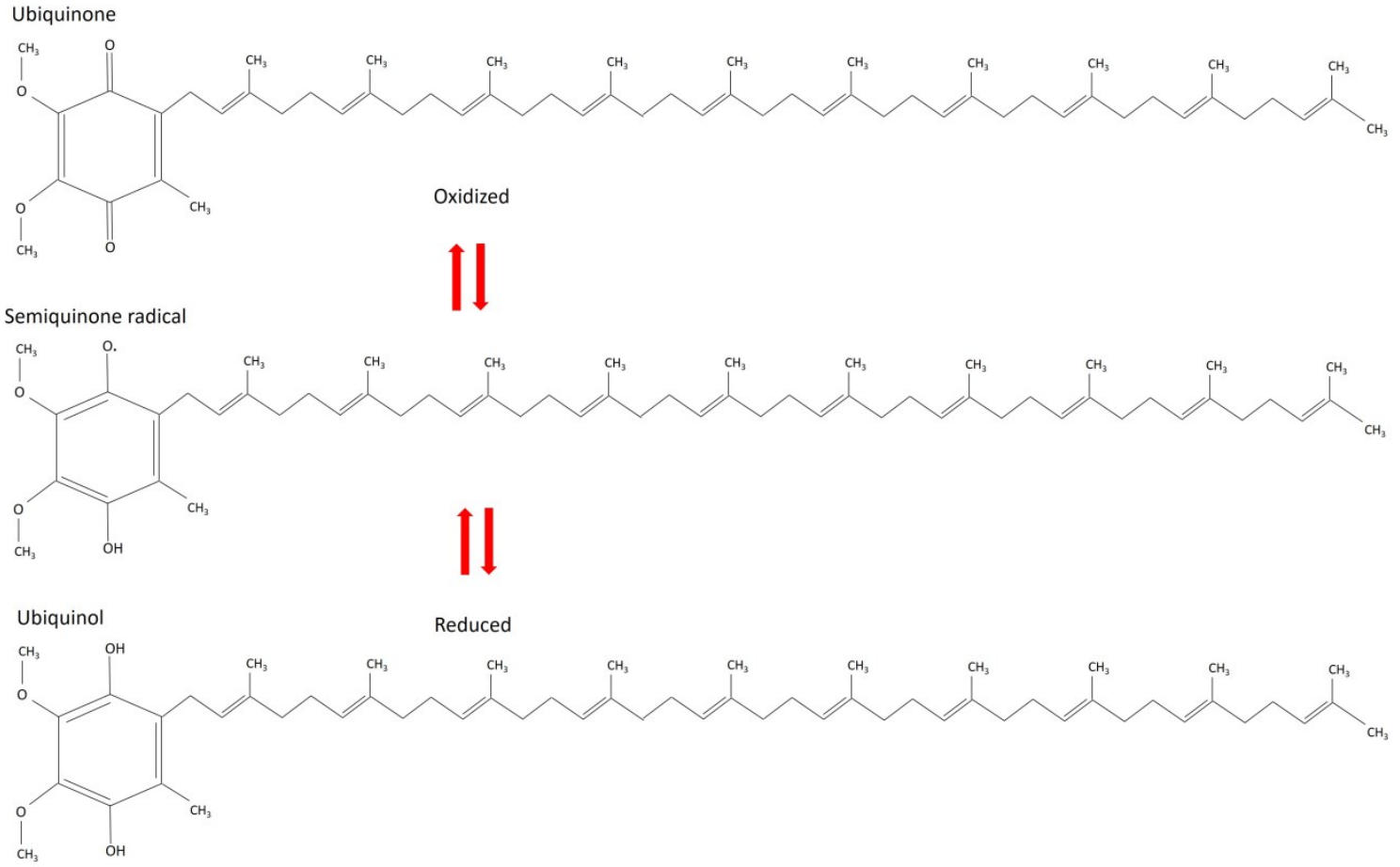

B

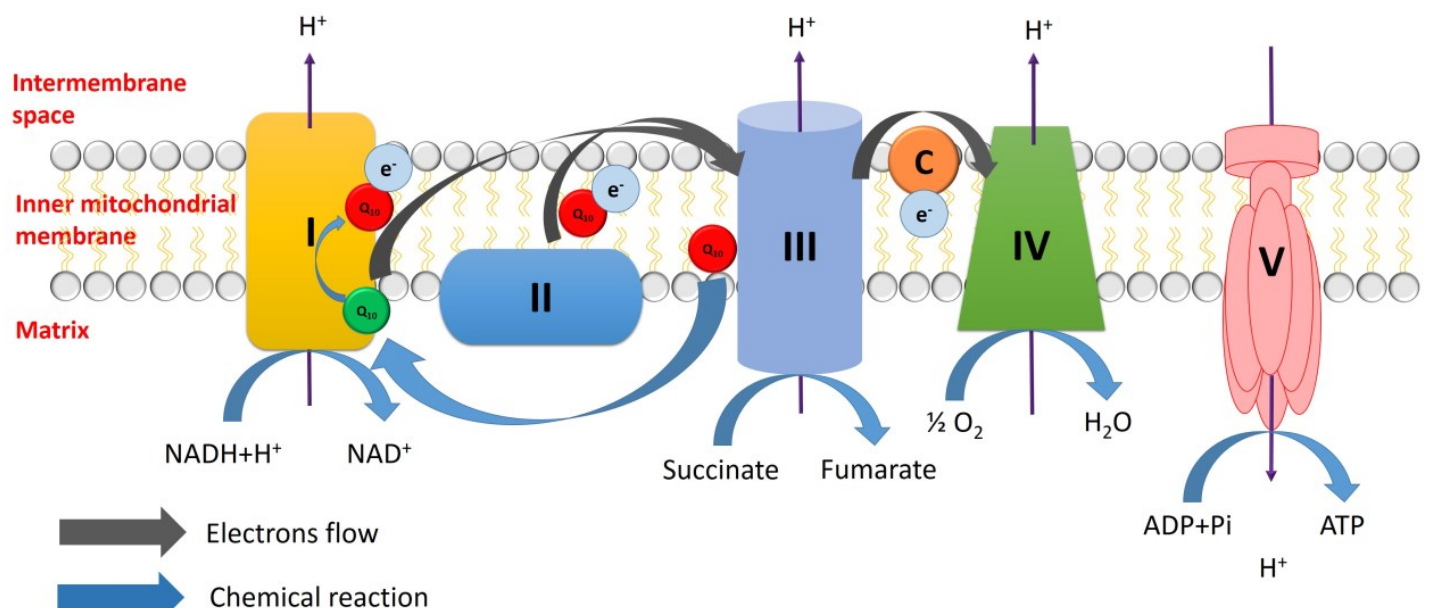

Chemical reaction

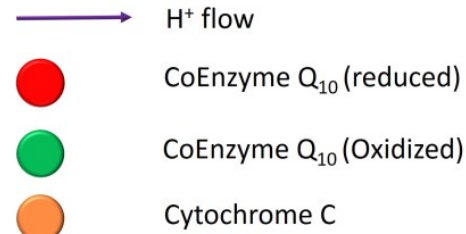

634

Cytochrome C

635

636

637

638 
Figure 2

A
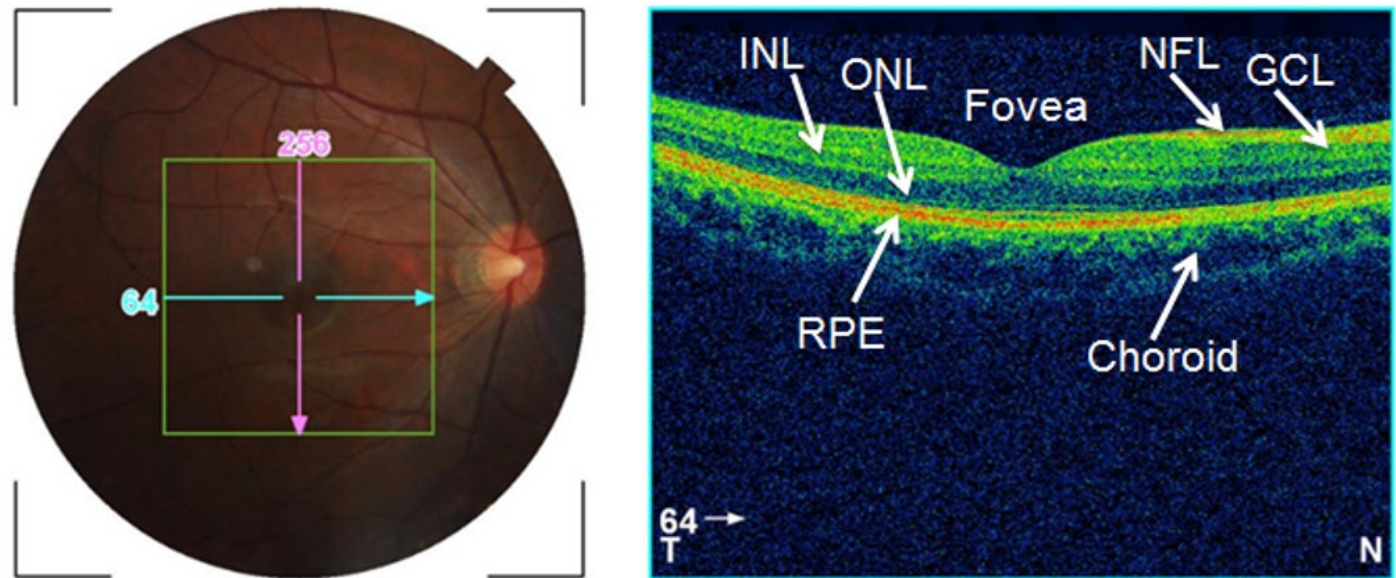

Retinal thickness map ILM - RPE / Red-free
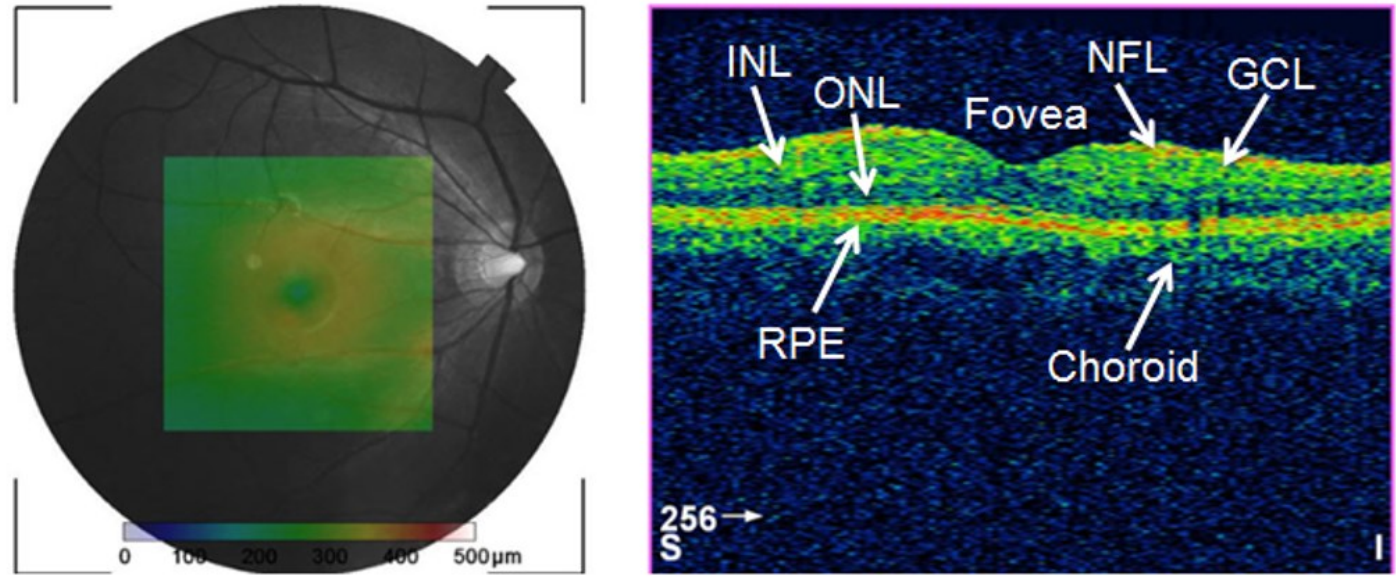

B
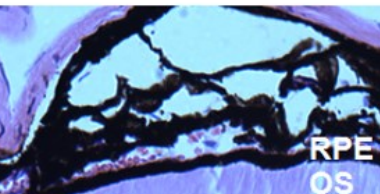

tons
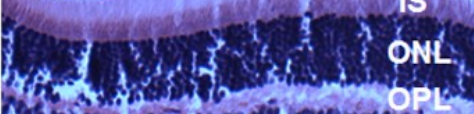

Q.1 3 .

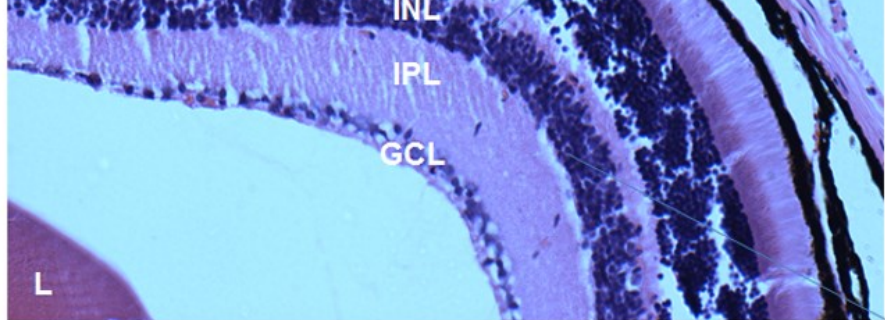

os

IS

ONL

OPL

INL 
$643 \quad$ Figure 3

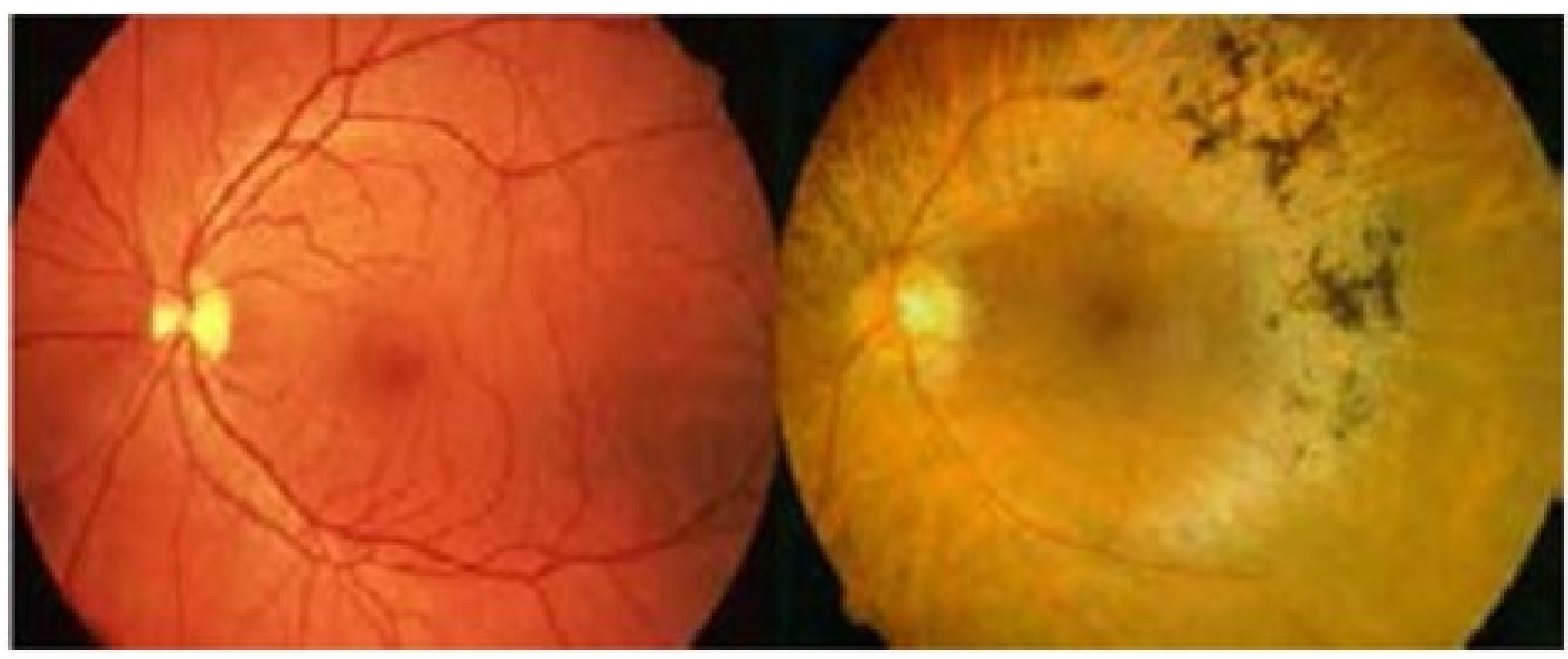

645

$646 \quad$ Figure 4
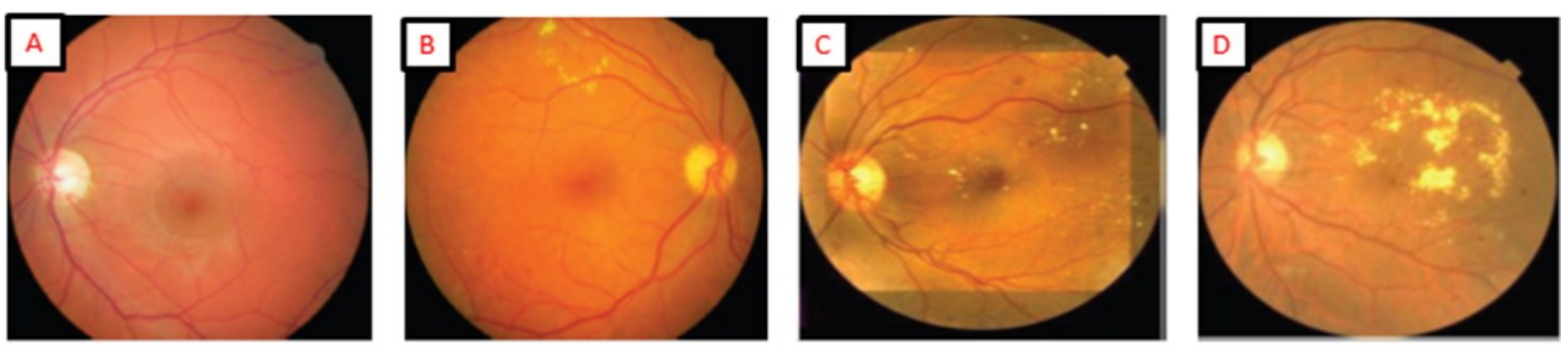

647
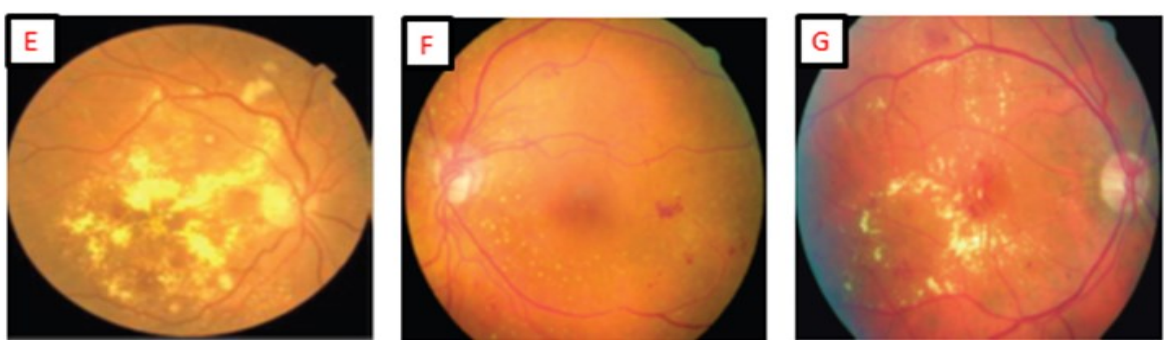

648

649

650

651

652

653

654

655 
656 Figure 5

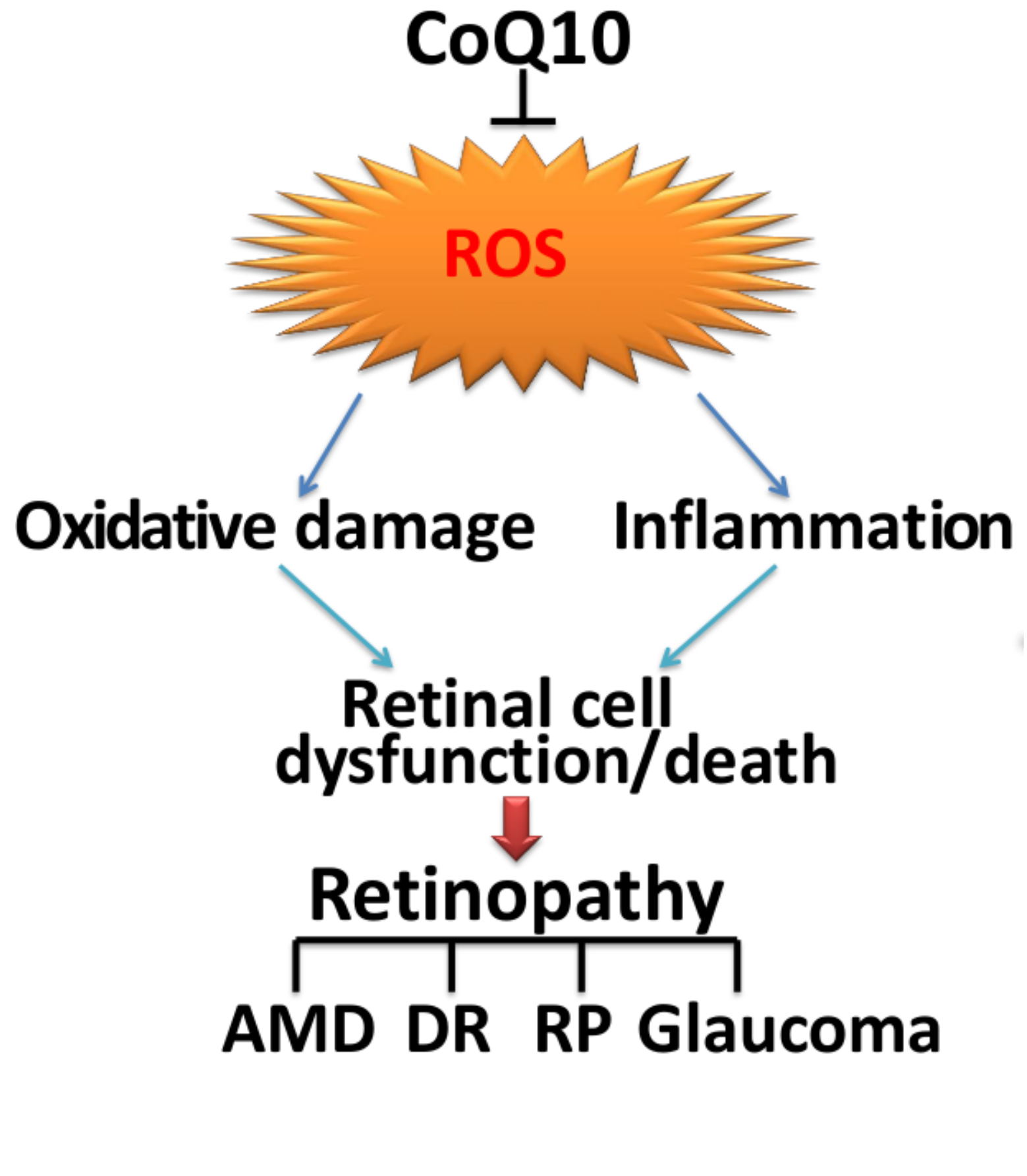

